\title{
Você conhece a nomenclatura biológica? Aprenda a forma correta de escrever os
}

\section{nomes dos organismos}

\author{
Do you know the biological nomenclature? Learn the correct way to write the names of organisms \\ ¿Conoces la nomenclatura biológica? Aprende la forma correcta de escribir los nombres de los \\ organismos
}

Recebido: 31/01/2022 | Revisado: 05/02/2022 | Aceito: 13/02/2022 | Publicado: 18/02/2022

Patrick de Castro Cantuária

ORCID: https://orcid.org/ 0000-0002-3676-7866 Instituto de Pesquisas Científicas e Tecnológicas do Estado do Amapá, Brasil E-mail: patrickcantuaria@gmail.com

Tonny David Santiago Medeiros

ORCID: https://orcid.org/ 0000-0002-0891-1584 Instituto de Pesquisas Científicas e Tecnológicas do Estado do Amapá, Brasil E-mail: tonnyiepa@gmail.com

Maryele Ferreira Cantuária

ORCID: https://orcid.org/0000-0003-1298-9197 Instituto Federal de Educação, Ciência e Tecnologia do Amapá, Brasil

E-mail: maryeleferreira@gmail.com

Alana Carine Sobrinho Soares

ORCID: https://orcid.org/0000-0003-0464-1908

Universidade Federal do Amapá, Brasil

E-mail: alanaufra@hotmail.com

Breno Marques da Silva e Silva

ORCID: https://orcid.org/0000-0003-0031-1450

Universidade do Estado do Amapá, Brasil

E-mail: breno.silva@ueap.edu.br

Sheylla Susan Moreira da Silva de Almeida

ORCID: https://orcid.org/0000-0002-7687-8288

Universidade Federal do Amapá, Brasil

E-mail: sheyllasusan@yahoo.com.br

Amauri Herbert Krahl

ORCID: https://orcid.org/0000-0001-7862-9624

Instituto Nacional de Pesquisas da Amazônia, Brasil

E-mail: amauri.her.krahl@gmail.com

Carlos Eduardo Costa-Campos

ORCID: https://orcid.org/0000-0001-5034-9268

Universidade Federal do Amapá, Brasil

E-mail: dududueducampos@gmail.com

Raullyan Borja Lima e Silva

ORCID: https://orcid.org/0000-0003-4104-5611

Instituto de Pesquisas Científicas e Tecnológicas do Estado do Amapá, Brasil

E-mail: raullyanborja@gmail.com

\begin{abstract}
Resumo
Atualmente, a nomenclatura dos seres vivos é regida por códigos internacionais que são construídos de forma coletiva, envolvendo a comunidade científica. Essas construções servem para organizar a nomenclatura do passado e estabilizar os nomes das espécies, sendo cada código é independente e autônomo. O uso adequado, o conhecimento e a consulta aos códigos tornam a utilização das nomenclaturas mais uniformes, evitando, assim, ambiguidades $\mathrm{O}$ objetivo do presente artigo é realizar uma síntese dos códigos nomenclaturais para promover uma explicação concisa e funcional sobre o uso correto das nomenclaturas biológicas e suas utilidades, uma vez que o uso adequado torna os trabalhos mais uniformes e sem ambiguidades. A metodologia para a composição de todas as informações referentes à grafia dos nomes dos organismos foi feita por consulta direta: Código Internacional de Nomenclatura para Algas, Fungos e Plantas; Código Internacional de Nomenclatura para Plantas Cultivadas; Código Internacional de Nomenclatura Fitossociológica; Código Internacional de Nomenclatura Zoológica; Código Internacional de Nomenclatura de Procariotos; Código Internacional de Classificação e Nomenclatura de Vírus; Código Internacional de Nomenclatura Filogenética. A ausência ou superficialidade de discussões nomenclaturais dos organismos vivos (ou extintos) nos cursos de graduação nas universidades brasileiras (existem poucas exceções) contribuem para o desconhecimento da existência dos códigos de nomenclatura. Apresentou-se exemplos e elucidações sobre o uso
\end{abstract}


correto das nomenclaturas, bem como a descrição de um status (não classificado), nova subdivisão e classe para as plantas.

Palavras-chave: Biologia; Códigos; Nomes científicos; Universalidade.

\begin{abstract}
Currently, the nomenclature of living beings is governed by international codes that are built collectively, involving the scientific community. These constructions serve to organize the nomenclature of the past and to stabilize the names of the species, with each code being independent and autonomous. Proper use, knowledge and consultation of codes make the use of nomenclatures more uniform, thus avoiding ambiguities and its uses, since proper use makes the work more uniform and unambiguous. The methodology for composing all the information referring to the spelling of the names of the organisms was made by direct consultation: International Code of Nomenclature for Algae, Fungi and Plants; International Code of Nomenclature for Cultivated Plants; International Code of Phytosociological Nomenclature; International Code of Zoological Nomenclature; International Code of Prokaryote Nomenclature; International Code of Classification and Nomenclature of Viruses; International Code of Phylogenetic Nomenclature. The absence or superficiality of nomenclatural discussions of living (or extinct) organisms in undergraduate courses at Brazilian universities (there are few exceptions) contributes to the ignorance of the existence of nomenclature codes. Examples and elucidations were presented on the correct use of nomenclatures, as well as the description of a new status (unclassified), new subdivision, and new class for plants.
\end{abstract}

Keywords: Biology; Codes; Scientific names; Universality.

\title{
Resumen
}

Actualmente, la nomenclatura de los seres vivos se rige por códigos internacionales que se construyen de manera colectiva, involucrando a la comunidad científica. Estas construcciones sirven para organizar la nomenclatura del pasado y estabilizar los nombres de las especies, siendo cada código independiente y autónomo. El uso adecuado, el conocimiento y la consulta de los códigos hacen más uniforme el uso de las nomenclaturas, evitando así las ambigüedades y sus usos, ya que el uso adecuado hace que el trabajo sea más uniforme e inequívoco. La metodología para componer toda la información referente a la ortografía de los nombres de los organismos se realizó por consulta directa: Código Internacional de Nomenclatura para Algas, Hongos y Plantas; Código Internacional de Nomenclatura para Plantas Cultivadas; Código Internacional de Nomenclatura Fitosociológica; Código Internacional de Nomenclatura Zoológica; Código Internacional de Nomenclatura de Procariontes; Código Internacional de Clasificación y Nomenclatura de Virus; Código Internacional de Nomenclatura Filogenética. La ausencia o superficialidad de discusiones nomenclaturales de organismos vivos (o extinguidos) en cursos de pregrado en universidades brasileñas (hay pocas excepciones) contribuye al desconocimiento de la existencia de códigos de nomenclatura. Se presentaron ejemplos y dilucidaciones sobre el uso correcto de nomenclaturas, así como la descripción de un nuevo estatus (no clasificado), nueva subdivisión y nueva clase para plantas.

Palabras clave: Biología; Códigos; Nombres científicos; Universalidad.

\section{Introdução}

Os sistemas nomenclaturais foram formados para organização de nomes e auxiliar a classificação adotada para alguma finalidade, seja científica, médica, química, física ou biológica. O atual sistema de nomenclatura binomial para seres vivos é derivado da exemplar obra Systema Naturae difundida por Lineu (Linnaei, 1735).

As nomenclaturas passaram por várias transformações, principalmente ao longo dos séculos XV a XVII (Winston, 1999), em decorrência do período em que a Europa começou a expandir seus domínios para porções extracontinentais e passou a registrar a fauna e flora regionais de ambientes até então pouco conhecidos no Ocidente.

Essa nomenclatura enfrentava uma barreira para a comunicação científica, uma vez que as frase-nomes que designavam as espécies eram um elemento que não facilitava a comunicação entre os pares. A exemplo disso, sempre que se precisava mencionar a espécie Orchis mascula L. (Linnaei, 1753) se usava a frase-nome: Orchis morio mas folijs maculatis B.C. Pin [=C.Bauhin] (Bauhin, 1623).

Apesar de se associar a nomenclatura binomial diretamente ligada a Lineu, essa invenção foi proposta por Gaspar Bauhin (Bauhin, 1623); no entanto, o seu uso não foi contínuo. Lineu foi quem usou primeiramente de forma consistente ao publicar os trabalhos Species Plantarum (Linnaei, 1753) e a 10 a edição de Systema Naturae (Linnaei, 1758). Com essa uniformização e constância, possibilitou que a comunidade científica organizasse a nomenclatura ao longo do tempo de forma gradativa e envolvendo os grupos biológicos de forma distinta. Esse processo de evolução nomenclatural aconteceu de forma 
paulatina, à medida que a comunidade científica passava a estudar os elementos da biodiversidade.

A classificação Aristotélica (Aristotelis, 1562) auxiliou Nicolas Lemery a desenvolver a clássica nomenclatura conhecida com os Reino Animal, Vegetal e Mineral (Lemery, 1675). Esse é um dos exemplos que são importantes para demonstrar sobre o início do processo evolutivo nas nomenclaturas biológicas.

As questões nomenclaturais são bem comuns para os que necessitam citar o uso de nomes de organismos como flora, fauna e funga, apesar de se observar muitas transgressões sobre a correta forma de apresentação dos nomes, seja por questões de inabilidade ou desconhecimento das regras envolvidas no processo, e como isso tem sido muito pouco discutido nas instituições brasileiras que chegam a ser despercebidas pela comunidade na mesma escala em que Santos et al. (2021) discutem a "cegueira botânica", aqui no caso seria a "cegueira bionomenclatural".

Atualmente, a nomenclatura dos seres vivos é regida por códigos internacionais que são construídos de forma coletiva, envolvendo a comunidade científica. Essas construções servem para organizar a nomenclatura do passado e estabilizar os nomes das espécies, sendo cada código independente e autônomo. Esses apresentam uma funcionalidade específica para determinar um conjunto de regras e recomendações para uniformização desses nomes associados às suas classificações vigentes. Desse modo, o objetivo do presente artigo é realizar uma síntese dos códigos nomenclaturais para promover uma explicação concisa e funcional sobre o uso correto das nomenclaturas biológicas e suas utilidades.

\section{Metodologia}

Para a composição de todas as informações referentes à grafia dos nomes dos organismos consultou-se todos os códigos nomenclaturais vigentes para confirmação, bem como trabalhos que versem sobre essas questões. Assim, os códigos são:

(a) Código Internacional de Nomenclatura para Algas, Fungos e Plantas (Turland et al., 2018) [sigla em inglês: CIN; Código de Shenzhen];

(b) Código Internacional de Nomenclatura para Plantas Cultivadas (Brickell, 2016) [sigla em inglês: ICNCP; Código de Plantas Cultivadas];

(c) Código Internacional de Nomenclatura Fitossociológica (Theurillat et al., 2020); [sigla em ingês: ICPN; Código Fitossociológico];

(d) Código Internacional de Nomenclatura Zoológica (1999) [sigla em inglês: ICNZ; Código Zoológico];

(e) Código Internacional de Nomenclatura de Procariotos (Parker et al., 2019) [Sigla em inglês: ICNP; Código Procariótico];

(f) Código Internacional de Classificação e Nomenclatura de Vírus (Lefkowitz et al., 2018; King et al., 2018) [sigla em inglês: ICVCN; Código de Vírus];

(g) Código Internacional de Nomenclatura Filogenética (Cantino \& Queiroz, 2020) [Sigla em inglês: Phylocode; Filocódigo].

\section{Resultados e Discussão}

A base nomenclatural da maioria dos códigos que regem os nomes biológicos é o latim. O seu uso não foi escolhido pela comunidade científica ao acaso, uma vez que, esse idioma é considerado uma língua franca, ou seja, idioma utilizado para comunicação, a qual foi amplamente integrada pelos eruditos entre os séculos XV à XVIII. Hoje, o inglês apresenta essa função para se estabelecer comunicação na comunidade científica internacional. Nas porções seguintes far-se-á elucidação do uso correto da nomenclatura com exemplos práticos. 
(a) Código Internacional de Nomenclatura para Algas, Fungos e Plantas (Turland et al., 2018)

Os nomes dos táxons de algas, fungos e plantas devem vir escritos somente com a primeira letra maiúscula e o restante minúsculo e em itálico, incluindo o nome genérico e nomes específicos ou epíteto, seguidos dos seus descritores. Em alguns casos, é desejável que seja apresentado a informação do protólogo em que a descrição ou diagnose validante foi publicada (Turland et al., 2018). A presença dos parênteses também deve ser observada, uma vez que é um elemento importante para que o leitor reconheça se o grupo já passou por mudança nomenclatural; no entanto, nem sempre esse elemento se configura como presente na nomenclatura.

Entre os nomes genéricos e específicos, pode haver termos de ligação como os designativos à subgênero, seção e série, mas é importante mencionar que as terminologias conectantes não fazem parte do nome da espécie. A explicação das regras está bem clara nos Artigos 21, 23, 26 e 46 do código em questão. Os nomes supragenéricos devem vir acompanhados, inicialmente, com o descritor e não são grafadas em itálico. É sempre interessante rever a forma de apresentação dos nomes, evitando-se o uso entre parênteses para qualquer hierarquia taxonômica, uma vez que esse elemento pode, em algum momento, estar presente na nomenclatura. Para os nomes dos descritores existe uma forma de apresentação indicada em Brumett e Powel (1992); no entanto, a lista atualizada é encontrada em International Plant Name Index (Ipni, 2022). As classificações superiores foram consultadas parcialmente em Brands (2022).

Sendo observado na sequência a seguir:

Mundo: Mundus Plinius Secundus (Plinii Secvndi, 1524)

Multiverso: Multiversus (William James, 1895) ex Cantuária stat. nov. (existentia plures mundus)

Universo: Universus Lucretius (Lucretius, 1495)

Astro: Astra Plinius Secundus (Plinii Secvndi, 1524)

Naturalia Aristotelis (Aristotelis, 1562)

Biota (Wagner, 2004) Wiemann, de Queiroz, Rowe, Planavsky, Anderson, Gogarten, Turner \& Gauthier, 2020

Superdomínio Neomura Caval.-Sm. 1987

Domínio Eucaryota Chatton 1925

Subdomímio Neokaryota Caval.-Sm. 1993

Infradomínio Neozoa Caval.-Sm. 1993

Supereino Corticata (Lankester, 1878) emend. Caval.-Sm. 2002

Reino: Plantae Haeckel 1866

Subreino Viridiplantae Caval.-Sm. 1981

Infrareino Streptophyta Jeffrey 1967

Superdivisão: Embryophyta Engler ex Pirani \& J. Prado 2018

Divisão Tracheophyta Sinnott ex Caval.-Sm. (1998)

Subdivisão Euphyllophytina (Kenrick \& P.R.Crane) ex Cantuária subd. nov. (folia megaphylla, eulophyta, anterozoides multiflagellata)

Classe Spermatopsida (Serbet \& G.W.Rothwell) ex Cantuária cl. nov. (vascularium cambium, fasciculi latioribus vinculis parenchymatis interfascicularis separati, plantis cum seminibus)

Subclasse: Magnoliidae Novák ex Takht. (1967)

Superordem: Lilianae Takht. (1967)

Ordem: Asparagales Link 1829

Família: Orchidaceae Juss. 1789 
Subfamília: Epidendroideae Lindl. ex Kostel. 1831

Tribo Malaxideae Lindl. 1826

Subtribo Dendrobiinae G.Don 1839

Gênero Bulbophyllum Thouars 1822

Espécie Bulbophyllum antoniobenjaminii Cantuária \& J.B.F.Silva 2020 (Cantuária et al. 2020)

Em se tratando de nomes infraespecíficos, van Steenis (1955) tem um excelente trabalho para elucidar sobre esse tipo de situação, inclusive evidenciando que, em florestas tropicais, onde tem alta diversidade de plantas, é mais difícil o botânico taxonomista observar a plasticidade encontrada nas populações, diferentemente do que é observado com mais facilidade em ambientes temperados; isso reflete diretamente no número de descrições infraespecíficas.

Ainda no trabalho de van Steenis (1955), são apontadas algumas recomendações para se trabalhar com essas categorias e, em especial, na recomendação de número 6, a qual menciona sobre hibridação (Kornet, 1990); aqui, em observação parcialmente contrária, não se pode deixar de mencionar que qualquer espécie descrita, um dia foi produto de alguma hibridação. Assim, faz-se a seguinte pergunta retórica: quem não é híbrido? A construção e organização de nomes botânicos são pensadas em uma sistematização que reflita na estabilidade. Para exemplificar a escrita de subespécie, variedade, forma, utiliza-se a configuração a seguir (Meneguzzo, 2020; World Flora On Line, 2022):

Batemannia colleyi subsp. peruviana (Mast.) Meneguzzo 2020 (Meneguzzo, 2020) [subespécie]

Coryanthes speciosa var. espiritosantensis Ruschi, 1975 [variedade]

Aganisia cyanea f. albiflora Meneguzzo \& Van den Berg 2015 (Meneguzzo et al., 2015) [forma]

No caso específico de híbridos naturais (nothoespécies), a grafia é feita incluindo o nome genérico adicionado do símbolo de multiplicação e do epíteto correspondente. O símbolo citado é semelhante a letra "x" minúscula; todavia, são elementos gráficos diferentes $(x \neq \mathrm{x})$ e no caso da ausência do símbolo de multiplicação em um sistema gráfico, é permitido o uso do "x" minúsculo como indicado no art. H.3A (Turland et al., 2018). Ver na sequência:

Catasetum $\times$ sheyllae Krahl, Cantuária \& J.B.F.Silva, 2021 (Cantuária et al., 2021) [nothoespécie/híbrido natural]

O Código de Shenzhen (Turland et al., 2018) também é soberano para nomes de algas e fungos, apresentando regras que se aplicam em alguns casos de forma específica para cada grupo. Consultas às bases de dados Index Fungorum (2022) e Algae Base (Guiry; Guiry, 2022) esclarecem sobre os descritores dos referidos grupos taxonômicos:

Uromyces amapaensis Sotão \& Piovezan, 2021 (Oliveira et al., 2021) [Fungo]

Bambusina borreri (Ralfs) Cleve var. majus C.B.Araújo, C.E.M.Bicudo \& S.Melo (Araújo et al., 2021) [Alga]

(b) Código Internacional de Nomenclatura para Plantas Cultivadas (BRICKELL et al. 2016)

Para se entender o funcionamento do ICNCP é importante conhecer o conceito da terminologia cultivar. De acordo com art. 2 uma cultivar é um conjunto de plantas que foram selecionadas para que um determinado caractere, ou combinação desses, apresente-se uniforme e estável e se for propagado, retém esse caráter (Brickell et al., 2016).

Os nomes de plantas cultivadas devem ser grafados indicando o gênero ou espécie seguido com aspas simples (") do epíteto, sendo importante lembrar que o nome do cultivar não é afetado pela troca do nome botânico. 
O nome do cultivar é escrito no idioma do autor, não é necessário fazer a tradução, e não deve ser escrito em itálico. O nome do cultivar só é valido quando publicado e registrado em catálogos nacionais e regionais, e podem apresentar característica de Cultivar, Grupo ou Enxerto-quimérico. Pode ser demonstrado nos trabalhos de Zhang et al. (2019) e Brickell et al., (2016):

\section{Narcissus ‘Alnwick Beauty’ D.C. MacArthur (Cultivar)}

Allium cepa Shallot Group (Grupo)

Citrus 'Hongrou Huyou' [Citrus changshan-huyou Y.B.Chang + Citrus unshiu (Swingle) Marcow.] (Enxertoquimérico)

É muito comum, encontrar problemas com uso correto da nomenclatura biológica correspondente ao Código de Plantas Cultivadas, principalmente envolvendo trabalhos na área de genética, ecologia ou de caráter revisional (Walker et al., 2006; Frank \& Chitwood, 2016).

(c) Código Internacional de Nomenclatura Fitossociológica (Theurillat et al., 2020); [sigla em ingês: ICPN]

Para iniciar a discussão sobre o ICPN, é necessário remeter ao conceito de Syntáxons, sendo unidades abstratas definidas por critérios florístico-sociológicos baseados em fitocenoses, juntamente com tipos de vegetação abstratos que ocupam habitats específicos delimitados em escalas espaciais finas que suportam comunidades criptogâmicas e fanerógamas, em especial epífitas (Theurillat et al., 2020).

Existem quatro principais categorias Syntaxonômicas sendo: Classe (classis=Cl.), Ordem (ordo=O.), Aliança (alliancia=Al.), Associação (associativo=As.), sendo grafadas da seguinte forma (Borhidi et al., 1979; Cano et al., 2009):

Cl. Chrysobalano-Annonetea glabrae Borhidi \& Muñiz in Borhidi, Muñiz \& Del-Risco 1979

O. Tabebuio-Bucidetalia (Lvov 1967) Borhidi \& Del-Risco in Borhidi, Muñiz \& Del-Risco 1979

Al. Marcgravio rubrae-Pterocarpion officinalis Cano (2009)

As. Roystoneo hispaniolanae-Pterocarpetum officinalis Cano (2009)

(d) Código Internacional de Nomenclatura Zoológica (1999)

A governança para nomes de animais é atribuída ao Código Internacional de Nomenclatura Zoológica (1999) que possui o objetivo de promover a estabilidade e universalidade dos nomes científicos de animais para que cada nome de táxon seja único e distinto (Papavero, 1994).

Apesar de existirem várias categorias taxonômicas (Reino, Filo, Classe, Ordem, Família, Gênero e Espécie) com suas supra e infra denominações, o Código Zoológico se propõe a regimentar sobre as três últimas categorizações. Todos os táxons supragenéricos são formados apenas por uma palavra latina e sem apresentação itálica e, quando possível, devem vir acompanhado do descritor e ano. O nome específico é formado por dois nomes (nome do gênero e nome da espécie) sendo apresentado em itálico ou sublinhado quando escrito manualmente, para que o leitor consiga reconhecer o destaque no texto. Quando apresentado pela primeira vez, é sempre interessante estar seguido do nome do descritor e ano da publicação da espécie. Ver a seguir: 
Subreino Bilateria Bilateria (Hatschek, 1888) Cavalier-Smith, 1983

Infrareino Deuterostomia Deuterostomia Grobben, 1908

Filo Chordata Bateson, 1885

Subfilo Vertebrata Cuvier, 1812

Infrafilo Gnathostomata Zittel, 1879

Superclasse Tetrapoda Goodrich, 1930

Classe Mammalia Linnaeus, 1758

Subclasse Theria (Parker \& Haswell, 1897) M.C. McKenna \& S.K. Bell, 1997

Infraclasse Eutheria Gill, 1872

Ordem Primates Linnaeus, 1758

Subordem Euprimates Hoffstetter, 1978

Infraordem Haplorhini (Pocock, 1918) M.C. McKenna \& S.K. Bell, 1997

Parvordem Anthropoidea (Mivart, 1864) M.C. McKenna \& S.K. Bell, 1997

Subparvordem Catarrhini É. Geoffroy Saint-Hilaire, 1812

Superfamilia Hominoidea Gray, 1825

Familia Hominidae Gray, 1825

Subfamilia Homininae Gray, 1825

Tribo Hominini Gray, 1825

Subtribo Hominina Gray, 1825

Gênero Homo Linnaeus, 1758

Espécie Homo sapiens Linnaeus, 1758

(e) Código Internacional de Nomenclatura de Procariotos (Parker et al., 2019)

Os nomes científicos atribuídos aos procariotos (exceto cianobactérias) apresentam nomenclatura binomial para nomes específicos; são construídos por palavras latinas designativas para gênero e espécie, escritas com a primeira letra em caixa alta seguida de letras minúsculas para o restante do binômio. Sendo os demais grupos taxonômicos grafados com uma única sentença nomenclatural. O uso de siglas fica estabelecido depois de ser descrito a primeira vez no texto de forma completa.

O Código Procariótico não reconhece o estrato infraespecífico de variedade, por considerar que sua conceituação está muita próxima da terminologia aplicada à subespécie (ver regra 5 c de Parker et al., 2019).

O Código Internacional de Nomenclatura de Procariotos é um instrumento de comunicação científica que apresenta a função basilar de regular a nomenclatura dos organismos regidos pelo presente código. Como observado:

Ordem Pseudomonadales Orla-Jensen 1921

Family Pseudomonadaceae Winslow et al. 1917

Gênero Pseudomonas Migula 1894

Subgênero Acetobacter (subgen. Gluconoacetobacter Yamada and Kondo 1985) liquefaciens (Asai 1935) Yamada and Kondo 1985.

Espécie Pseudomonas thermotolerans Manaia \& Moore 2002

Subespécie Bacillus subtilis subsp. spizizenii Nakamura et al. 1999. 
(f) Código Internacional de Classificação e Nomenclatura de Vírus (LEFKOWITZ et al., 2018; KING et al., 2018)

A organização do ICVCN é de competência do Comitê Internacional de Taxonomia de Vírus (ICTV) que pertence à Divisão de Virologia da União Internacional de Sociedades Microbiológicas (UISM). Apresenta governança para nomes de vírus, viróides e ácidos nucléicos satélites, sendo totalmente independente das demais nomenclaturas biológicas, tanto que, na propositura do BioCódigo, é vista como uma exceção.

A forma de apresentação do nome dos vírus é bem diferente das outras nomenclaturas. Os nomes das espécies virais são escritos em itálico, com a primeira palavra começando com uma letra maiúscula, as outras palavras só se apresentam com letra maiúsculas se forem substantivos próprios ou identificadores alfabéticos. Um nome de espécie não deve ser abreviado. O ICTV editou um arquivo bastante claro denominado "Como escrever vírus, espécies e outros nomes de táxons” (Ictv, 2020), e nele é indicado que os nomes de vírus nunca devem ser abreviados.

Algo bastante controverso para a maioria dos pesquisadores (exceto virologistas) é a forma de escrita dos nomes de vírus e das espécies, que são apresentados em formatos diferentes; o nome de vírus sempre vem escrito de forma regular (não itálico) enquanto das espécies ou de outras categorias taxonômicas são em itálico (para facilitar o entendimento, a primeira exemplificação será aplicada em uma frase):

Os isolados de dengue vírus 4 foram obtidos da espécie Dengue virus, que pertence à categoria taxonômica "Realm", por não possuir uma palavra que semanticamente tenha uma tradução que não se confunda com o clado inferior de Reino, então pertencente ao Domínio (Realm) Riboviria e ao Reino Orthornavirae.

A base do ICVCN é o inglês e o latim (categorias superiores) e, para conhecer a forma correta da escrita das categorias taxonômicas, sempre está associada à validação dos nomes, que passam por um comitê; este recebe as propostas via formulário específico. Para exemplificar sobre as autorias dos táxons será incluído os autores dos formulários como responsáveis como autores dos nomes:

Domínio "Realm": Riboviria Gorbalenya et al. (2017)

Reino: Orthornavirae Koonin et al. (2019)

Filo: Pisuviricota Koonin et al. (2019)

Classe: Pisoniviricetes Koonin et al. (2019)

Ordem: Nidovirales (Pringle, 1996) movido por Gorbalenya et al. (2017) movido por Koonin et al. (2019)

Subordem: Cornidovirineae Brinton et al. (2017) movido por Gorbalenya et al. (2017) movido por Koonin et al.

(2019)

Família: Coronaviridae Wildy, 1971 [1975] movido por Pringle (1996) Brinton et al. (2017) movido por Gorbalenya et al. (2017) movido por Koonin et al. (2019)

Subfamília: Orthocoronavirinae Groot et al. (2008) movido por Brinton et al. (2017) movido por Gorbalenya et al. (2017) movido por Koonin et al. (2019)

Gênero: Betacoronavirus Groot et al. (2008) movido por Brinton et al. (2017) movido por Gorbalenya et al. (2017) movido por Koonin et al. (2019)

Subgênero: Sarbecovirus Ziebuhr (2017) movido por Gorbalenya et al. (2017) movido por Koonin et al. (2019)

Espécie: Severe acute respiratory syndrome-related coronavirus ICTV 2003[2004] movido por Groot et al. (2008) movido por Gorbalenya et al. (2017)

Um nome coletivo para um grupo de vírus pertencentes a um táxon de nível superior não deve ser apresentado em itálico nem em maiúsculas: 
A dengue virose ou dengue ou vírus dengue é causada pelo Dengue virus.

Para viróides e ácidos nucléicos satélites que também são de governança do ICVCN seguem as formas corretas de aplicação:

Família Pospiviroidae

Gênero Pospiviroid

Espécie Chrysanthemum stunt viroid

Família Tolecusatellitidae

Gênero Betasatellite

Espécie: Cotton leaf curl Kashmir betasatellite

(g) Código Internacional de Nomenclatura Filogenética (Cantino \& Queiroz, 2020)

O Filocódigo é uma propositura de classificação científica baseada nos relacionamentos filogenéticos de ancestralidades dos grupos biológicos, sendo embasado na sistemática filogenética. Os nomes dos clados são estabelecidos pela conversão dos nomes de grupos biológicos existentes na literatura científica.

Outra questão bastante relevante para entender no presente código é o fato das categorias taxonômicas não constituírem parte integrante do processo de nomenclatura filogenética, segue a exemplificação:

Antestrombus Maxwell, Dekkers, Rymer,

Congdon \& Maxwell, 2020

[S. Maxwell, 2021]

nomen cladi conversum

\section{Considerações Finais}

A ausência ou superficialidade de discussões nomenclaturais dos organismos vivos (ou extintos) nos cursos de graduação nas universidades brasileiras (existem poucas exceções) contribuem para o desconhecimento da existência dos códigos de nomenclatura. Apesar de existir propostas de unificação das nomenclaturas, como o caso do Biocódigo, esse é um assunto que necessita de maior debate para que se tenha uma maior aplicabilidade e conhecimento sobre como consultar os códigos vigentes.

Em trabalhos futuros serão abordados situações concretas específicas de cada grupo biológico para que o pesquisador ou estudantes possam consultar os nomes de forma simples e intuitiva.

\section{Agradecimentos}

Ao Instituto de Pesquisas Científicas e Tecnológicas do Estado do Amapá, Universidade Federal do Amapá e Universidade do Estado do Amapá.

\section{Referências}


Araújo, C. B., Bicudo, C. E. M., Takiyama, L. R., \& Melo, S. (2021). Pseudofilamentous desmids (Zygnematophyceae) from an Amazonian floodplain lake (Macapá, AP, Brazil). Porto Alegre, Iheringia, Série Botânica, 75. https://isb.emnuvens.com.br/iheringia/article/view/764/498

Borhidi, A., Muñiz, O., \& Del-Risco, E. (1979). Clasificación fitocenológica de la vegetación de Cuba. Acta Botanica Academiae Scientiarum Hungaricae, Budapest, 25: 263-301. https://www.biodiversitylibrary.org/part/162156

Brands, S. J. (ed.), 2022. The Taxonomicon. Universal Taxonomic Services, Zwaag, The Netherlands. [http://taxonomicon.taxonomy.n1/].

Brickell, C. D., Alexander, C., Cubey, J. J., David, J. C., Hoffman, M. H. A., Leslie, A. C., Malécot, V., \& Jin, X. (2016). International Code of Nomenclature for Cultivated Plants. Scripta Horticulturae (9th ed.). International Society of Horticultural Science. 18.

Brinton, M. A., Gulyaeva, A., Balasuriya, U. B. R., Dunowska, M., Faaberg, K. S., Goldberg, T., Leung, F. C.-C., Nauwynck, H. J., Snijder, E. J., Stadejek, T., \& Gorbalenya, A. E. (2017). Expansion of the rank structure of the family Arteriviridae and renaming its taxa. Form. 2017.012S. https://talk.ictvonline.org/taxonomy/p/taxonomy-history?taxnode_id=202006105.

Brummitt, R. K., \& Powell, C. E. (1992). Authors of plant names. A list of authors of scientific names of plants, with recommended standard form of their names including abbreviations. Royal Botanic Gardens, Kew: 1992. (4)732.

Cano, E., Veloz, A. R., Cano-Ortiz, A., \& Esteban. F. J. R. (2009) Analysis of the Pterocarpus officinalis forests in the Gran Estero (Dominican Republic). Acta Botanica Gallica, 156(4). 559-570. https://www.tandfonline.com/toc/tabg20/156/4.

Cantuária, P. C., Silva, J. B. F., Farias, J. E., Silva, D. A. S., \& Medeiros, T. D. S. (2020). Uma nova espécie de Bulbophyllum Thouars (Orchidaceae Juss.) para a Amazônia Brasileira. Biota Amazônia, 10, 36-38.

Cantuária, P. C., Krahl, D. R. P., Krahl, A. H., Chiron, G., \& Silva, J. B. F. (2021). Catasetum × sheyllae (Orchidaceae: Catasetinae), a new natural hybrid from Brazilian Amazon. Phytotaxa, 527(4), 257-265. https://www.biotaxa.org/Phytotaxa/article/view/phytotaxa.527.4.3.

Cavalier-Smith, T. (1981). The origin and early evolution of the eukaryotic cell. In: Carlile, M. J., Collins, J. F., \& Moseley, B. E. B. (eds.). Molecular and Cellular Aspects of Microbial Evolution. 32 nd. Symposium of the Society for General Microbiology. Cambridge University Press. 33-84. https://socgenmicrobiol.org.uk/pubs/symposia.cfm.

Cavalier-Smith, T. (1987). The origin of eukaryote and archaebacterial cells. Annals of the New York Academy of Sciences, 503, 17-54. https://nyaspubs.onlinelibrary.wiley.com/doi/epdf/10.1111/j.1749-6632.1987.tb40596.x.

Cavalier-Smith, T. (1993). Percolozoa and the symbiotic origin of the metakaryote cell. In: Ishikawa, H., Ishida, M., Sato, S. (orgs.) Endocytobiology. Tübingen University Press. 399-406.

Cavalier-Smith, T. (1998). A revised six-kingdom system of life. London, Biological Reviews, 73, p. 203-266. https://pubmed.ncbi.nlm.nih.gov/9809012/

Cavalier-Smith, T. (2002). The phagotrophic origin of eukaryotes and phylogenetic classification of Protozoa. International Journal of Systematic and Evolutionary Microbiology. London, 52, 297-354. https://www.microbiologyresearch.org/content/journal/ijsem/10.1099/00207713-52-2-297

Chatton, É. Pansporella perplexa, Amoebien a spores protegees parasite des Daphnies. Reflexions sur la biologie et la phylogenie des Protozoaires. Annales des Sciences Naturelles Zoologie. Paris, ser. 10e serie, t. VII. 1925. p.1-84. https://eurekamag.com/research/023/326/023326569.php.

Cronquist, A., Takhtajan, A., \& Zimmermann, W. (1966). On the Higher Taxa of Embryobionta. Taxon. Utrecht, 15(4). https://www.iapttaxon.org/historic/Congress/IBC_1969/embryobionta.pdf.

Don, G. (1839). SubTribe II. Dendrobieae. In: Sweet, R. Sweet's Hortus Britannicus: Or, A Catalogue of All the Plants Indigenous Or Cultivated in the Gardens of Great Britain, Arranged According to the Natural System, with the Generic and Specific Names, English Names, Accentuation, Derivation of Generic Names, Native Country, year of introduction, Time of Flowering, Colour of Flowers, Mode of Growth, Duraction, References to the Best Figures, The Most Usefull Synomymes, The the Linnean Class and Order to which Each Genus Belongs, \& $\mathrm{c}$ https://play.google.com/books/reader?id=xP4CAAAAYAAJ\&pg=GBS.PP1\&hl+=pt

Franck, M. H., \& Chitwood, D. H. (2016). Plant chimeras: The good, the bad, and the'Bizzaria'. Developmental Biology, Rockville, 419, 41-53. https://www.sciencedirect.com/science/article/pii/S0012160616300902?via\%3Dihub\#f0010.

Gorbalenya, A. E., Krupovic, M., Siddell, S., Varsani, A., \& Kuhn, J. H. (2017). Riboviria: establishing a single taxon that comprises RNA viruses at the basal rank of virus taxonomy. Form 2017.006G. https://talk.ictvonline.org/taxonomy/p/taxonomy-history?taxnode_id=202007095.

Groot, R. J., Ziebuhr, J., Poon, L. L., Woo, P. C., Talbot, P. R., Rottier, P. J. M., Holmes, K. V., Baric, R., Perlman, S., Enjuanes, L., \& Gorbalenya, A. E. (2008). Revision of the family Coronaviridae. Form 2008.085-126V. https://talk.ictvonline.org/ictv/proposals/2008.085-122V.v4.Coronaviridae.pdf.

Guiry, M. D., \& Guiry, G. M. (2022). AlgaeBase. World-wide electronic publication, National University of Ireland, Galway. https://www.algaebase.org/.

Haeckel, E. (1866). Generelle Morphologie der Organismen. Berlin, G. Reimer. https://doi.org/10.5962/bhl.title.3953.

International Commission on Zoological Nomenclature. (1999). International code of zoological nomenclature, 4th ed. International Trust for Zoological Nomenclature. Editorial Committee. W.D.L. Ride, - Chairman, H.G. Cogger, C. Dupuis, O. Kraus, A. Minelli, F. C. Thompson, P.K. Tubbs. https://code.iczn.org/summary/?frame $=1$.

IPNI (2022). International Plant Names Index. Published on the Internet. http://www.ipni.org, The Royal Botanic Gardens, Kew, Harvard University Herbaria \& Libraries and Australian National Botanic Gardens.

Index Fungorum. (2022). http://www.indexfungorum.org/ 
James, W. (1895). Is Life Worth Living? International Journal of Ethics. Chicago, 6(1). https://www.jstor.org/journal/intejethi.

Jeffrey, C. (1967). The origin and differentiation of the Archegoniate land plants: A second contribution". Kew Bulletin. London, $21(2), 335349$. https://www.jstor.org/stable/4108533

Jussieu, A. L. (1753). Genera Plantarum: secundum ordines naturales disposita, juxta methodum in Horto regio parisiensi exaratam, anno M.DCC.LXXIV. 1789. Pl.: 64. 4 Aug. (Orchideae), nom. cons. Validated by a description in Latin. - T: Orchis L. (1753). https://www.biodiversitylibrary.org/item/7125\#page/159/mode/1up.

Kenrick, P., \& Crane, P. R. (1997). The Origin and Early Diversification of Land Plants: A Cladistic Study. Washington: Smithsonian Institution Press. 441 p.

Koonin, E. V., Dolja, V. V., Krupovic, M., Varsani, A., Wolf, Y. I., Yutin, N., Zerbini, M., \& Kuhn, J. H. (2019). Create a megataxonomic framework, filling all principal taxonomic ranks, for realm Riboviria. Form 2019.006G. https://talk.ictvonline.org/taxonomy/p/taxonomy-history?taxnode_id=202007198.

King, A. M. Q., Lefkowitz, E. J., Mushegian, A. R., Adams, M. J., Dutilh, B. E., Gorbalenya, A. E., Harrach, B., Harrison, L. R., Junglen, S., Knowles, N. J., Kropinski, A. M., Krupovic, M., Kuhn, J. H., Nibert, M. L., Rubino, L., Sabanadzovic, S., Sanfaçon, H., Siddell, S. G., Simmonds, P., Varsani, A., Zerbini, F. M., Murilo, F., \& Davison, A. J. (2018). Changes to taxonomy and the International Code of Virus Classification and Nomenclature ratified by the International Committee on Taxonomy of Viruses. Archives of Virology. Switzerland, 163, p. $2601-2631$. https://link.springer.com/article/10.1007\%2Fs00705-018-3847-1\#citeas.

Kornet, D. J. On specific and infraspecific delimitation. (1990). In: Baas, P., Kalkman, K., Geesink, R. (eds). The Plant Diversity of Malesia. Springer, Dordrecht. https://link.springer.com/chapter/10.1007/978-94-009-2107-8_30.

Kosteletzky, V. F. (1831). Allgemeine medizinisch-pharmazeutische Flora: enthaltend die systematische Aufzählung und Beschreibung sämmtlicher bis jetzt bekannt gewordenen Gewächse aller Welttheile in ihrer Beziehung auf Diätetik, Therapie und Pharmazie, nach den natürlichen Familien des Gewächsreiches geordnet. Pl. 233, v. 4. https://books.google.com.br/books/about/Allgemeine_medizinisch_pharmazeutische_F.html?id=E4kfAAAAYAAJ\&redir_esc=y.

Lankester, E. R. Preface. (1878). In Gegenbaur, C. Grundriss der vergleichenden Anatomie. London: Macmillan, (2a ed.), https://trove.nla.gov.au/work/11305094.

Lefkowitz, E. J., Dempsey, D. M., Hendrickson, R. C., Orton, R. J., Siddell, S. G., \& Smith, D. B. (2018). Virus taxonomy: the database of the International Committee on Taxonomy of Viruses (ICTV). England: Oxford University Press, Nucleic acids research, v. 46. https://talk.ictvonline.org/.

Lemery, N. (1675). Cours de Chymie contenant la maniere de faire les operations qui sont en usage dans la medecine, par une methode facile avec des raisonnements chaque operation, pour l'instruction de ceux qui veulent s'appliquer a cette science. Paris. https://gallica.bnf.fr/ark:/12148/bpt6k739985/f3.item\#.

Lindley, J. (1821-1826). Collectanea botanica, or, Figures and botanical illustrations of rare and curious exotic plants. 8(7). https://www.biodiversitylibrary.org/item/27998\#page/101/mode/1up.

von LINK, J. H. F. (1829). Handbuch zur Erkennung der Nutzbarsten und am Haufigsten Vorkommenden Gewächse. Berlin. https://www.biodiversitylibrary.org/page/53334725\#page/282/mode/1up.

Linnaei, C. (1735). Sveci, Doctoris Medicinae systema naturae, sive, Regna tria naturae systematice proposita per classes, ordines, genera, \& species. Lugduni Batavorum [Leiden, the Netherlands]:Apud Theodorum Haak :Ex Typographia Joannis Wilhelmi de Groot. https://www.biodiversitylibrary.org/bibliography/877.

Linnaei, C. (1753). Species plantarum: exhibentes plantas rite cognitas ad genera relatas, cum diferentiis specificis, nominibus trivialibus, synonymis selectis, locis natalibus, secundum systema sexuale digestas. Cum privilegio S. R. Mais Sueciae \& S. R. Polonicae ae Electoris Saxon. Holmiae, Impemsis Lauremtii Salvii. https://www.biodiversitylibrary.org/bibliography/37656.

Linnaei, C. (1753). Systema Naturae per regna tria naturae, secundum classes, ordines, genera, species cum characterabus differentiis, synomymis, locis. $10^{\mathrm{a}}$. Cum privilegio S. $\quad$ R. Mais Sueciae. Holmiae, Impemsis $\quad$ Lauremtii https://upload.wikimedia.org/wikipedia/commons/a/a2/Sistema_Naturae_\%281758\%29.pdf.

Lucretius, T. C. T. (1495). Lucretii Cari De rerum natura libri primus. https://www.google.com.br/books/edition/Bibl iotheca_Spenceriana_Or_a_Descriptive/k0AqtiBo71gC?hl=ptBR\&gbpv=1\&dq=Lucretius+De+rerum+natura+liber+primus+1495\&pg=PA155\&printsec=front cover.

Maxwell, S. J., Dekkers, A. M., Rymer, T. L., \& Congdon, B. C. (2020). Towards Resolving the American and West African Strombidae (Mollusca: Gastropoda: Neostromboidae) Using Integrated Taxonomy. The Festivus, San Diego, 52(1), 3-38, https://www.thesandiegoshellclub.com/uploads/1/3/8/1/138179831/festivus_53_3_august_2021-pages_192_maxwell.pdf.

Maxwell, S. J. (2021). Registration of Neostromboidae Clades in the RegNum of the PhyloCode, and Errata. The Festivus, San Diego, 53(3), 192-209. https://www.thesandiegoshellclub.com/uploads/1/3/8/1/138179831/festivus_52_1_february_2020-pages-4-39.pdf.

Meneguzzo, T. E. C. (2020). Taxonomic and nomenclatural notes on Zygopetalinae infraspecies (Orchidaceae). Heringeriana, Brasília, 14(2), 157-191. http://revistas.jardimbotanico.ibict.br/index.php/heringeriana/article/view/917939.

Meneguzzo, T. E. C., Baumgratz, J. F. A., \& Van Den Berg, C. (2015). Taxonomic studies in the Aganisia complex (Orchidaceae, Zygopetalinae). Phytotaxa: a rapid international journal for accelerating the publication of botanical taxonomy, 238, 1-39. https://www.mapress.com/phytotaxa/content/2015/f/p00238p039f.pdf.

Oliveira, P. M. P., Sotão, H. M. P., Freire, G. S. F., \& Monteiro, J. S. (2021). Two new species of rust fungi (Pucciniales) from the Brazilian Amazon: Aecidium margaritariae on Margaritaria and Uromyces amapaensis on Jatropha. Acta Amazonica, 51(3), $244 \quad-\quad 249$. https://www.scielo.br/j/aa/a/bGG5qZWzxv3rCg7YjGTzNks/?format=pdf\&lang=en. 
Papavero, N. (1994). Fundamentos práticos de taxonomia zoológica: Coleções, bibliografia, nomenclatura. (2a ed.), Editora da UNESP \& FAPESP, 185p.

Parker, C. T., Tindall, B. J., \& Garrity, G. M. (2019). International Code of Nomenclature of Prokaryotes. International Journal of Systematic and Evolutionary Microbiology, Englad, n. 69. https://www.microbiologyresearch.org/content/journal/ijsem/10.1099/ijsem.0.000778.

Pirani, J. R., \& Prado, J. P. (2018). Embryopsida, a new name for the class of land plants. Bratislava, Taxon, 61(5). https://onlinelibrary.wiley.com/doi/abs/10.1002/tax.615014.

Plinii Secvndi, C. (1524). Naturalis Historiae, novicium Camenis Quiritium tuorum opus, natos apud me proxima fetura licentiore epistula narrare constitui tibi, iucundissime Imperator, sit enim haec tui praefatio, verissima, dum maximi consenescit in patre. namque tu solebas nugas esse aliquid meas putare, ut obiter emolliam Catullum conterraneum meum (agnoscis et hoc castrense verbum): ille enim, ut scis, permutatis prioribus syllabis duriusculum se fecit quam volebat $\quad$ existimari $\quad$ a $\quad$ Veraniolis $\quad$ suis $\quad$ et https://play.google.com/store/books/details/Gaius_Plinius_Secundus_Naturalis_historiae_Opus_a?id=YUxXAAAAcAAJ.

Pringle, C. R. (1996). Virus Taxonomy 1996. A Bulletin from the Xth International Congress of Virology in Jerusalem. London, Archives of Virology, 141, 2251-2256. Virology Division News. https://link.springer.com/article/10.1007/BF01718231.

Ruschi, A. (1975). Coryanthes speciosa var. Espiritosantensis, Ruschi, n. var. Boletim do Museu de Biologia Prof. Mello Leitão. Série Botânica, Santa Tereza, 81(1), 1-4. http://boletim.sambio.org.br/pdf/01_02.pdf.

Santos, M. I., Pontes, A. N., \& Martins Junior, A. S. (2021). Percepção de docentes de biologia sobre a presença da "cegueira botânica" em escolas públicas do Estado do Pará. Research, Society and Development, 10(13), e216101321106. 10.33448/rsd-v10i13.21106 https://rsdjournal.org/index.php/rsd/article/view/21106.

van Steenis, C.G.G.J. (1955). Specific and infraspecific delimitation. Flora Malesiana, Series 1, Spermatophyta, Edinburgo, 5(1), 167-234. https://repository.naturalis.nl/pub/532674.

Takhtajan, A. L. (1966, mas publicado em 04 fev. 1967). Sistema i Filogeniia Tsvetkovykh Rastenii (Systema et Phylogenia Magnoliophytorum), Soviet Sciences Press, Leningrad. Nauka, Moscow, 611, 1967. https://books.google.com.br/books?id=xljPEon9Qy0C\&printsec=frontcover\&hl=pt$\mathrm{BR} \&$ source=gbs_ge_summary_r\&cad=0\#v=onepage\&q\&f=false.

Theurillat, J., Willner, W., Fernández-González, F., Bültmann, H., Čarni, A., Gigante, D., Mucina, L., \& Weber, H. (2020). International Code of Phytosociological Nomenclature. 4th edition. Applied Vegetation Science. 24(1). https://onlinelibrary.wiley.com/doi/10.1111/avsc.12491.

Thouars, L. M. A. D. P. (1822). Histoire particulière des plantes orchidées recueillies dans les trois îles australes de d'Afrique, de France, de Bourbon et de Madagascar. L'auteur, Arthus Bertrand, Treuttleret Wurtz, Paris. https://www.biodiversitylibrary.org/item/9881\#page/3/mode/1up.

Turland, N. J., Wiersema, J. H., Barrie, F. R., Greuter, W., Hawksworth, D. L., Herendeen, P. S., Knapp, S., Kusber, W.H., Li, D. Z., Marhold, K., May, T. W., Mcneill, J., Monro, A. M., Prado, J., Price, M. J., \& Smith, G. F. (eds.) [2018]. International Code of Nomenclatura for algae, fungi, and plants (Shenzhen Code) adopted by the Nineteenth International Botanical Congress Shenzhen, China, July 2017. Regnum Vegetabile 159. Glasshütten: Koeltz Botanical Books.

Walker, A. R., Lee, E., \& Robinson, S. P. (2006). Two new grape cultivars, bud sports of Cabernet Sauvignon bearing pale-coloured berries, are the result of deletion of two regulatory genes of the berry colour locus. Plant Molecular Biology, 62, 623-635. https://link.springer.com/article/10.1007\%2Fs11103-0069043-9.

Wiemann, J., de Queiroz, K., Rowe, T. B., Planavsky, N. J., Anderson, R. P. Gogarten, J. P., Turner, P. E., Gauthier, J. A. (2020). Biota Wagner, 2004: converted clade name. In: de Queiroz, K., Cantino, P. D., Gauthier, J. A. (eds.). Phylonyms: A Companion to the PhyloCode. CRC Press, Boca Raton, Florida. 1324 pp. https://www.taylorfrancis.com/books/mono/10.1201/9780429446276/phylonyms-kevin-de-queiroz-philip-cantino-jacques-gauthier?refId=3012f028c044-4f0b-ad6c-3a4ee27c6492\&context=ubx.

Wildy, N. P. L. (1971-1975). Classification and Nomenclature of Viruses. 1st Report of the International Committee on Nomenclature of Viruses. Birmingham. https://www.karger.com/Book/Home/218074.

Winston, J. E. (2000). Describing Species: Practical Taxonomic Procedure for Biologists. Columbia University Press.

Word Flora on line. 2022. Published on the Internet. http://www.worldfloraonline.org/.

Zhang, M. Jing, L., Wu, Q., Zhu, K., Ke, F., Xu, J., Zhao, S., Wang, G., \& Zhang, C. (2019). Metabolite profile comparison of a graft chimera 'Hongrou Huyou' (Citrus changshan-huyou + Citrus unshiu) and its two donor plants. BMC Plant Biology, 582(19). https://www.ncbi.nlm.nih.gov/pmc/articles/PMC6933880/.

Ziebuhr, J., Baric, R. S., Baker, S., DE Groot, R. J., Drosten, C., Gulyaeva, A., Haagmans, B. L., Neuman, B. W., Perlman, S., Poon, L. L. M., Sola, I., \& Gorbalenya, A. E. (2017). Reorganization of the family Coronaviridae into two families, Coronaviridae (including the current subfamily Coronavirinae and the new subfamily Letovirinae) and the new family Tobaniviridae (accommodating the current subfamily Torovirinae and three other subfamilies), revision of the genus rank structure and introduction of a new subgenus rank. Form 2017.013S. https://talk.ictvonline.org/taxonomy/p/taxonomyhistory?taxnode_id=202006129. 